\title{
RETRACTED ARTICLE: Preparation/characterization of solid dispersions and enhancement of dissolution rate on celecoxib as BCS II class
}

\author{
Jung Hwan Lee Young Lae Kim · So Jin Lee · Jaewon Yang • \\ Jin Young Park $\cdot$ Eun Young Kim $\cdot$ Dongwon Lee $\cdot$ Gilson Khang
}

Received: 14 April 2013/Accepted: 12 August 2013/Published online: 12 September 2013

(C) The Korean Society of Pharmaceutical Sciences and Technology 2013

The article has been retracted upon request of the first author since significant portions of the article were published earlier in the following article: Jung Hwan Lee, Min Jeong Kim, Hyeon Yoon, Cho Rok Shim, Hyun Ah Ko, Sun Ah Cho, Dongwon Lee, Gilson Khang (2013)
Enhanced dissolution rate of celecoxib using PVP and/or HPMC based solid dispersions prepared by spray drying method, Journal of Pharmaceutical Investigation 43:205-213 DOI 10.1007/s40005-013-0067-2.

J. H. Lee $\cdot$ Y. L. Kim $\cdot$ S. J. Lee $\cdot$ J. Yang

J. Y. Park · E. Y. Kim · D. Lee · G. Khang $(\bowtie)$

Department of BIN Fusion Technology, Department of Polymer

Nano Science \& Technology and Polymer BIN Research Center,

Chonbuk National University, 567 Baekje-daero, Deokjin,

Chonju 561-756, Korea

e-mail: gskhang@chonbuk.ac.kr; gskhang@jbnu.ac.kr 